EPJ Web of Conferences 43, 02004 (2013)

DOI: $10.1051 /$ epjconf/20134302004

(C) Owned by the authors, published by EDP Sciences, 2013

\title{
The galactic globular cluster M5 (NGC 5904) as a particle physics laboratory
}

\author{
N. Viaux $1,2,3,4, a$, M. Catelan ${ }^{1,2,3}$, G.G. Raffelt ${ }^{4}$, J. Redondo ${ }^{4}$ \\ and A.A.R. Valcarce ${ }^{5}$ \\ ${ }^{1}$ Departamento de Astronomía y Astrofísica, Pontificia Universidad Católica de Chile, \\ Av. Vicuña Mackenna 4860, 782-0436 Macul, Santiago, Chile \\ 2 The Milky Way Millennium Nucleus, Av. Vicuña Mackenna 4860, 782-0436 Macul, Santiago, \\ Chile \\ ${ }^{3}$ Pontificia Universidad Católica de Chile, Centro de Astroingeniería, Av. Vicuña Mackena \\ 4860, 782-0436 Macul, Santiago, Chile \\ ${ }^{4}$ Max-Planck-Institut für Physik (Werner-Heisenberg-Institut), Föhringer Ring 6, \\ 80805 München, Germany \\ ${ }^{5}$ Universidade Federal do Rio Grande do Norte, Depto. de Física, \\ 59072-970 Natal, RN, Brazil
}

\begin{abstract}
Globular clusters have been used for a long time to test stellar evolution theories, and in particular to constrain novel forms of energy loss in low-mass stars. This includes constraints on axion properties, neutrino dipole moments, milli-charged particles, Kaluza-Klein gravitons, and many other phenomena. Depending on their interaction strength, these particles can be abundantly produced in stellar interiors, escape without further interaction, and thus drain energy directly from the stellar interior. Hence, they contribute directly to the stellar energy losses, thus modifying stellar evolution. Our goal is to re-examine such constraints in the light of modern data and updated stellar evolution codes, paying particular attention to systematic and statistical errors. As a first example, we consider the case of a neutrino magnetic moment that enhances the energy loss from the plasma process.

In terms of the observed color-magnitude diagrams, the tip of the red giant branch (RGB) has been identified as a sensitive observable of the effects of the energy losses due to a neutrino magnetic moment. Here we describe the consequences of adding the cooling effect due to a neutrino magnetic moment to the Princeton-Goddard-PUC (PGPUC) stellar evolution code, exploring in particular the dependence of the position of the RGB tip on the neutrino magnetic moment.

As a first application, we studied the position of the observed RGB tip in the case of the Galactic globular cluster M5 (NGC 5904), using the latest, high-precision, ground-based data from the P. B. Stetson database (2012, priv. comm.). We compare the empirical results with the PGPUC model predictions, and discuss the implied constraints on the value of the neutrino magnetic moment.
\end{abstract}

\section{INTRODUCTION}

One of the oldest and most successful theories in astrophysics is stellar evolution. In particular, the latter is largely in agreement with observations of globular clusters (GCs; e.g., [16], [8]), which are stellar systems that may harbour up to several million stars. Thus, one can include new physical ingredients in stellar evolution calculations and compare the results with GC observations, leading to constraints on the new physics phenomena under investigation. For example, the properties of weakly interacting

\footnotetext{
ae-mail: nviaux@astro.puc.cl
}

This is an Open Access article distributed under the terms of the Creative Commons Attribution License 2.0, which permits unrestricted use, distribution, and reproduction in any medium, provided the original work is properly cited. 
particles such as axions or novel neutrino properties such as a neutrino magnetic moment (NMM) can be studied in this way. Indeed, GCs are considered one of the largest available laboratories for such particle physics studies (e.g., [11]).

In the present work, we carry out a comparison between stellar evolution predictions and highprecision observations of the GC M5 (NGC 5904), with an aim to study the NMM.

\section{EXOTIC ENERGY LOSS}

Novel neutrino properties (such as a milli-charge, dipole moment, etc.) enhance the thermal neutrino emission rates [2]. For instance, for a non-relativistic plasma, the energy loss rate per unit volume of a single neutrino species in plasmon decay processes $\gamma \rightarrow \bar{v} \nu$ is [11]:

$$
Q_{\gamma \rightarrow \nu \bar{v}} \approx \frac{8 \zeta_{3}}{3 \pi} T^{3} \times \begin{cases}\frac{C_{V}^{2} G_{F}^{2}}{\alpha}\left(\frac{\omega_{p l}^{2}}{4 \pi}\right)^{3} & \text { (standard model) }, \\ \alpha_{\nu}\left(\frac{\omega_{p l}^{2}}{4 \pi}\right) & \text { (milli-charge), } \\ \frac{\mu_{v}^{2}}{2}\left(\frac{\omega_{p l}^{2}}{4 \pi}\right)^{2} & \text { (dipole moment) },\end{cases}
$$

where $\zeta_{3}=1.202, \alpha_{v}=e_{v}^{2} / 4 \pi$, with $e_{v}$ the neutrino charge, $\mu_{v}$ is the magnetic dipole moment, $G_{F}$ is the Fermi constant, and $C_{V}$ is the effective neutral-current neutrino-electron vector-coupling constant.

The produced neutrinos drain energy out of the star, cooling the core of a RGB star before helium ignition. The changes in stellar evolution that leave the most noticeable imprints in the color-magnitude diagram (CMD) are (e.g., [10] [11] [4]):

- A delay of the helium flash: as a consequence of the additional cooling, the RGB star is able to evolve past the "classical" point for He ignition in the core. As a consequence, the core mass at helium ignition $\left(\mathcal{M}_{c}\right)$ increases, and therefore the luminosity of the tip of the RGB (TRGB) also increases.

- Due to the increment in $\mathcal{M}_{c}$, stars in the subsequent horizontal branch (HB) phase become more luminous as well, and therefore the HB lifetime also decreases.

In addition to neutrinos, one may also include other weakly interacting particles (like the axion) and derive constraints from stellar evolution (e.g., [5]). Close to the TRGB, the dominant process to create axions is the bremsstrahlung process, $e+Z e \rightarrow Z e+e+a$, due to the degenerate conditions in the helium core (e.g., [13]).

\section{OBSERVATIONAL FRAMEWORK}

We use the latest, high-precision, ground-based data from P. B. Stetson's database (2012, priv. comm.). The derived $I, V-I$ CMD is shown in Fig. 1 (left panel). This CMD is clearly affected by the presence of a large number of field interlopers. To remove the latter, we perform a statistical decontamination, using the method described in [6]. The cleaned CMD is shown in Fig. 1 (right panel).

In order to avoid uncertainties related to the distance and the interstellar extinction, we first estimate the difference in I-band magnitude between the TRGB and the so-called RGB luminosity function (LF) "bump," $\Delta I_{\mathrm{Bump}}^{\mathrm{TRGB}}$. The level of the bump in the M5 CMD was calculated on the basis of a differential $I$-band LF, and then fitting a Gaussian to the relevant feature. This gives $I_{\text {Bump }}=14.00 \pm 0.03 \mathrm{mag}$.

Before finding the TRGB, we need to distinguish between the RGB and asymptotic giant branch (AGB) components in M5. For that purpose we use a Yale-Yonsei $\left(\mathrm{Y}^{2}\right)$ isochrone [7] with $Y=0.245$ and $Z=0.00136$ (as appropriate for a GC such as M5), for an age of $12 \mathrm{Gyr}$, to assist us in tracing the RGB position. To transform these models to the observational plane, we use the empirical color transformations of [17]. We then compute the distance $\Delta$ (in mag units) of each star above the HB to the 
Ageing Low Mass Stars: From Red Giants to White Dwarfs

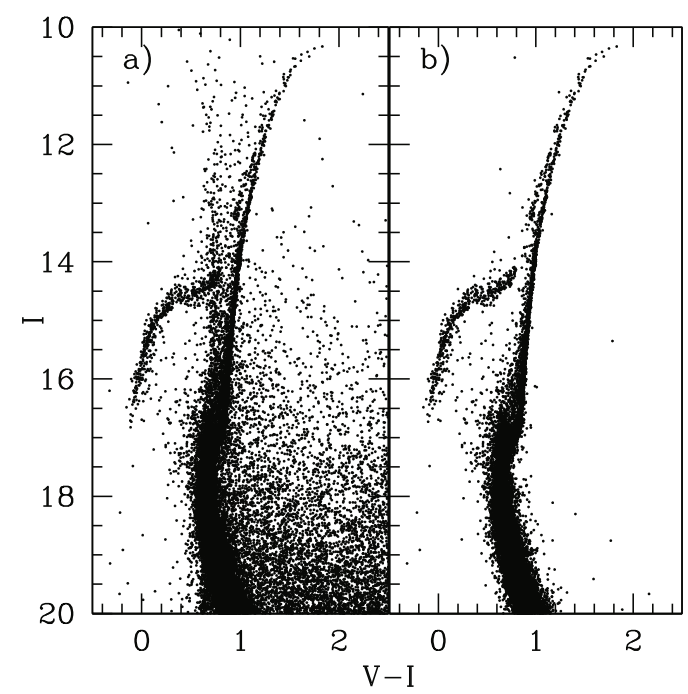

Figure 1. Left: Original M5 CMD; right: M5 CMD after field star decontamination.
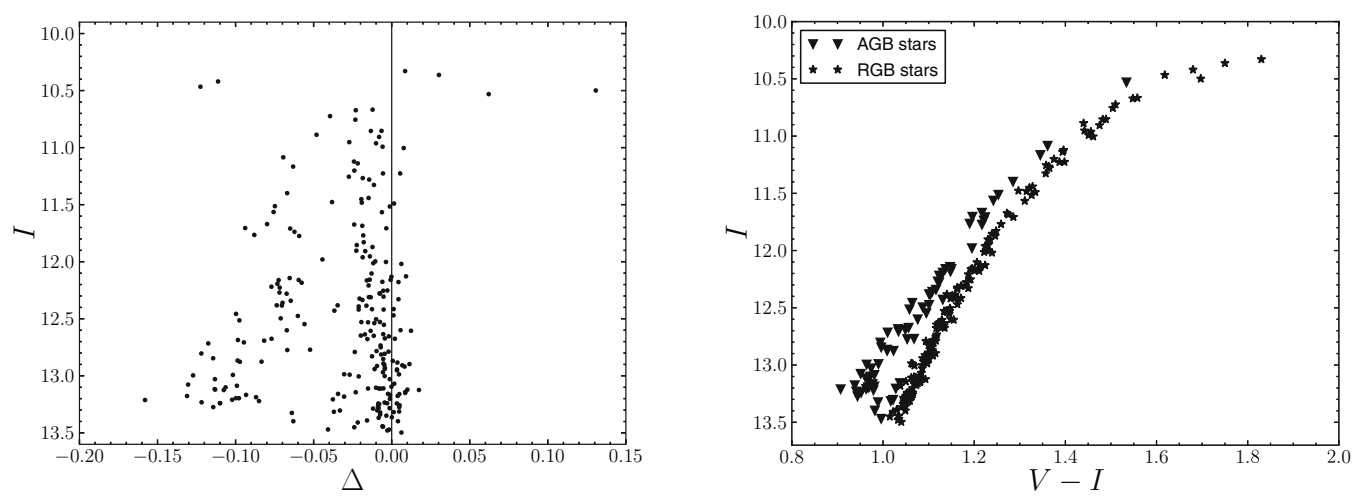

Figure 2. Left panel: Distance $\Delta$ (in mag units) of each star from above the HB level in the M5 CMD to the relevant isochrone, shown as a function of the $I$-band magnitude. Right panel: The resulting $I, V-I \mathrm{CMD}$, with AGB stars plotted as triangles and RGB ones as five-pointed stars.

isochrone. The resulting distance vs. I-band magnitude distribution is shown in Figure 2 (left panel). Clearly we see two groups. This mixed RGB/AGB sample is then separated thanks to EMMIX [9], which provides us with the most likely members of each of the two groups, as shown in Figure 2 (right panel).

To estimate the TRGB position in the M5 CMD we use the method of [10]. To assess the efficiency of this method we performed Monte-Carlo simulations using synthetic CMDs (SCMDs). Comparing the thus inferred TRGB $I$-band magnitude with the real value (Fig. 3), we found the statistical error of the [10] method to be $\sim 0.008 \mathrm{mag}$, with a negative bias of $0.01 \mathrm{mag}$, which can be easily corrected for.

\section{THEORETICAL FRAMEWORK}

To evaluate the impact of a NMM upon the evolutionary properties of low-mass stars, we use the PGPUC code [15], and include the NMM as described in [12]. This way, we obtain evolutionary tracks for 


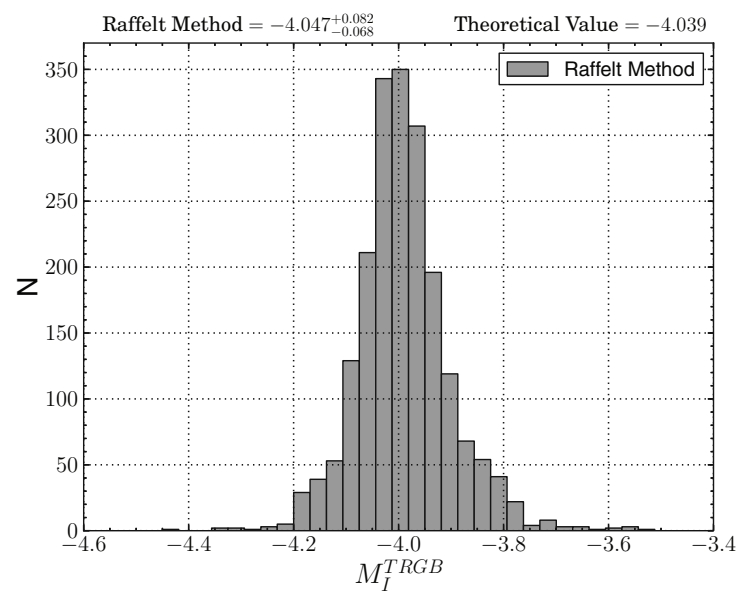

Figure 3. Histogram of the [10] method-based estimate of the TRGB $I$-band magnitude, as calculated for SCMDs reproducing M5.
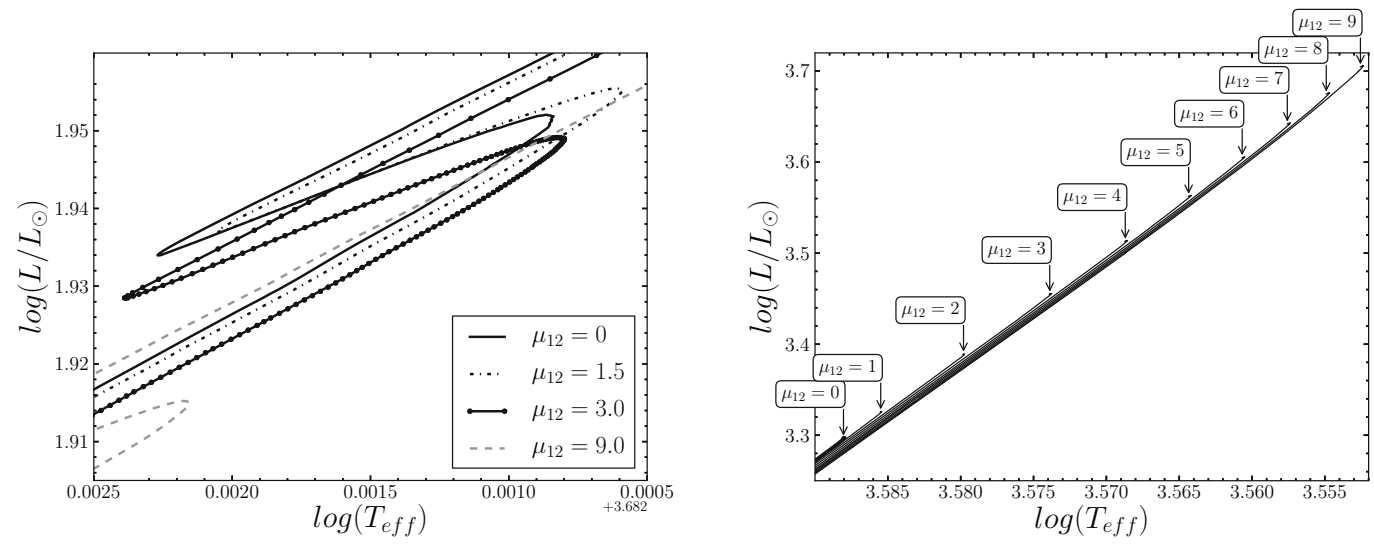

Figure 4. PGPUC evolutionary tracks around the RGB bump (left panel) and TRGB (right panel), for the different values of $\mu_{v}$ indicated in the insets. Here we use $\mu_{12}=\frac{\mu_{v}}{10^{-12} \mu_{B}}$.

different NMM values, covering the range $\mu_{v}=(0-9) \times 10^{-12} \mu_{B}$, where $\mu_{B}$ is the Bohr magneton. We use an initial mass of $0.82 M_{\odot}$ (representative of a RGB star, for the $Y$ and $Z$ values listed in Section 3 and an age of $12.9 \mathrm{Gyr}$ ). The computed evolutionary tracks for different $\mu_{v}$ values are shown in Figure 4.

Based on the computed tracks, we then evaluate the theoretically expected TRGB and RGB bump $I$-band magnitudes, and finally derive the predicted dependence of $\Delta I_{\mathrm{Bump}}^{\mathrm{TRGB}}$ on $\mu_{v}$. This is shown as filled circles in Figure 5. The estimated $1 \sigma$ error level (see Section 6 below) is indicated as error bars in this plot, and the $2 \sigma$ level as five-pointed stars. The next section compares these results with the observations.

\section{CONFRONTING THE OBSERVATIONAL RESULT WITH THEORETICAL PREDICTIONS}

The predicted $\Delta I_{\mathrm{Bump}}^{\mathrm{TRGB}}$ vs. $\mu_{v}$ relation is shown as a thick dashed line in Figure 5. Based on the observed M5 CMD (Fig. 1), the empirically derived $\Delta I_{\mathrm{Bump}}^{\mathrm{TRGB}}$ value is constrained to fall within the range of the 


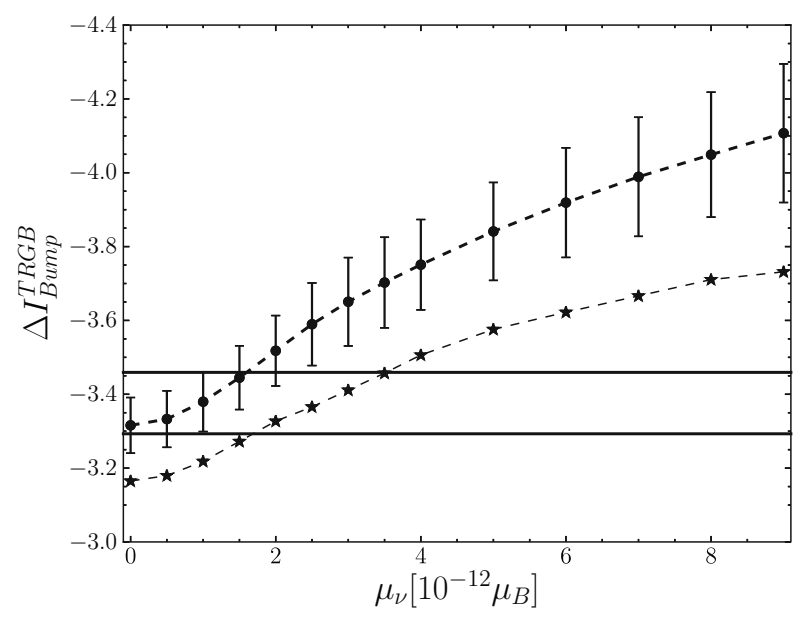

Figure 5. Comparison between predicted and observed $\Delta I_{\mathrm{Bump}}^{\mathrm{TRGB}}$ values. The predicted values are shown as filled circles with $1 \sigma$ error bars, and are plotted as a function of $\mu_{v}$. The five-pointed stars indicate a $2 \sigma$ deviation away from the filled circles. The horizontal lines constrain the empirically allowed $\Delta I_{\mathrm{Bump}}^{\mathrm{TRGB}}$ values.

Table 1. Uncertainties in the predicted TRGB position.

\begin{tabular}{|l|l|l|}
\hline Input parameter & Range & $\max \left(\left|\Delta M_{I}^{\mathrm{TRGB}}\right|\right)$ \\
\hline$Y$ & $0.230-0.250$ & 0.001 \\
\hline$Z$ & $0.0012-0.0014$ & 0.008 \\
\hline Mixing-length parameter $\alpha_{\mathrm{MLT}}$ & \pm 0.1 & 0.027 \\
\hline Atmospheric $T(\tau)$ relation & $\pm 5 \%$ & 0.007 \\
\hline${ }^{4} \mathrm{He}+{ }^{4} \mathrm{He} \rightarrow{ }^{8} \mathrm{Be}+\gamma$ reaction rate & $\pm 10 \%$ & 0.015 \\
\hline Thermal neutrino emission & $\pm 5 \%$ & 0.014 \\
\hline Conductive opacities & $\pm 10 \%$ & 0.017 \\
\hline RGB mass loss & $0-0.21 M_{\odot}$ & 0.032 \\
\hline Bolometric corrections $\left(\mu_{12}=0\right)$ & & 0.034 \\
\hline Bolometric corrections $\left(\mu_{12}=9\right)$ & & 0.192 \\
\hline
\end{tabular}

horizontal lines shown in this figure. This shows that, for $\mu_{v}>3.5 \times 10^{-12} \mu_{B}$, it is not possible for the theoretical TRGB to match the observations, at the $2 \sigma$ level.

\section{UNCERTAINTIES}

Uncertainties are key elements in the derivation of upper limits on non-standard physical parameters, and so we need to control all relevant systematic and statistical effects that can modify our derived constraints on $\mu_{\nu}$. In Table 1 we list some of the most important identified sources of uncertainties affecting the predicted TRGB position (column 1), with the corresponding estimated range of values (column 2) and how they propagate into the $I$-band magnitude of the TRGB (column 3, which actually shows the maximum encountered deviation from the reference case, $\left.\max \left[\left|\Delta M_{I}^{\mathrm{TRGB}}\right|\right]\right)$. This table shows that, in addition to bolometric corrections, the most important sources of uncertainty come from RGB mass loss, the mixing-length parameter $\alpha_{\text {MLT }}$ of mixing-length theory (MLT), conductive opacities, the triple$\alpha$ reaction rate, and the neutrino cooling rate. On the other hand, as for the $T(\tau)$ (temperature-optical 


\section{EPJ Web of Conferences}

depth) relation, Figure 7 of [14] reveals that a $\pm 5 \%$ uncertainty encompasses the different available prescriptions, but Table 1 shows that this has only a small impact on the predicted TRGB position. The remaining physical ingredients listed in Table 1 will be discussed further in Viaux et al. (2012, in preparation).

\section{CONCLUSIONS}

Updated stellar evolution codes are available and new observational data are in our hands. It is thus possible to update the constraint on the NMM and make an educated study of the possible sources of uncertainties. Here we estimate that a change by 0.03 mag in the $I$-band magnitude of the TRGB will change the constraint by $\approx 0.2 \times 10^{-12} \mu_{B}$. So from Figure 5 we conclude that $\mu_{v}<3.5 \times 10^{-12} \mu_{B}$ is a reasonable limit based on the present approach, which is significantly different from previous works on the subject, largely based on [10]. Still, our new result is in agreement with the similar estimates previously made by [10], [12], and [4]. In this regard, it is worth noting that these astrophysical constraints are at least one order of magnitude more stringent than available laboratory experiments (e.g., [1]).

The agreement with previous works on the subject notwithstanding, it is important to note that our analysis is based on a single GC, and so an extension to an enlarged sample of GCs would be important and indeed is planned for the near future. Even more important, however, is the fact that we have used the position of the RGB bump as a reference point in our analysis. Unfortunately, the predicted RGB bump position is known to be affected by ill-constrained physical processes, and is thus subject to large systematic errors (e.g., [3], and references therein). Such errors were not taken into account in the present study. In a future work, we plan to adopt different reference points, such as the HB level, in order to alleviate these problems.

We warmly thank P. B. Stetson for providing data in advance of publication. Support for N.V. and M.C. is provided by the Chilean Ministry for the Economy, Development, and Tourism's Programa Iniciativa Científica Milenio through grant P07-021-F, awarded to The Milky Way Millennium Nucleus; by Proyecto Fondecyt Regular \#1110326; by the BASAL Center for Astrophysics and Associated Technologies (PFB-06); and by Proyecto Anillo ACT-86. Support for N.V is also provided by MECESUP Project No. PUC0609 (Chile).

\section{References}

[1] A. G. Beda, V. B. Brudanin, V. G. Egorov, D. V. Medvedev, M. V. Shirchenko, A. S. Starostin, Phys. Part. Nucl. Lett., 7, 406 (2010).

[2] J. Bernstein, M. Ruderman, G. Feinberg, Phys. Part. Nucl. Lett., 7, 406 (1963).

[3] S. Cassisi, in Red Giants as Probes of the Structure and Evolution of the Milky Way, Ap\&SS Proceedings (Springer-Verlag: Berlin), p. 57 (2012).

[4] M. Catelan, J. A. de Freitas Pacheco, J. E. Horvath, ApJ, 461, 231 (1996).

[5] J. A. Frieman, S. Dimopoulos, M. S. Turner, Phys. Rev. D, 36, 2201 (1987).

[6] C. Gallart, et al., AJ, 125, 742 (2003).

[7] Y. C. Kim, P. Demarque, S. Yi, D. Alexander, ApJ, 143, 449 (2002).

[8] A. Marín-Franch, et al., ApJ, 694, 1498 (2009).

[9] G. J. McLachlan, D. Peel, J. Stat. Soft., 4, 1 (1999).

[10] G. G. Raffelt, ApJ, 365, 559 (1990). (R90)

[11] G. G. Raffelt, Stars as Laboratories for Fundamental Physics (Univ. of Chicago Press, 1996).

[12] G. G. Raffelt, A. Weiss, A\&A, 264, 536 (1992). (RW92) 
Ageing Low Mass Stars: From Red Giants to White Dwarfs

[13] G. G. Raffelt, A. Weiss, Phys. Rev. D, 51, 1495 (1995).

[14] J. Tanner, S. Basu, P. Demarque, preprint (arXiv:1209.3784, 2012).

[15] A. Valcarce, M. Catelan, A. V. Sweigart, A\&A, in press (arXiv:1208.5127, 2012). (VCS12)

[16] D. A. VandenBerg, ApJS, 129, 315 (2000).

[17] G. Worthey, H.-c. Lee, ApJ, 193, 1 (2011). 